\title{
Neumoencefalo a tensión tras herida en cuero cabelludo en portadora de válvula de derivación ventrículo-peritoneal: caso clínico y revisión de la literatura
}

\author{
L.G. González-Bonet; F.A. Goig-Revert; R. Rodríguez-Mena y C. Barcia-Mariño
}

Servicio de Neurocirugía. Hospital General Universitario de Valencia

\section{Resumen}

El neumoencéfalo se produce la mayoría de las veces por una solución de continuidad en el cráneo e incluso en las meninges. En ocasiones, ciertos factores pueden hacer que éste adopte las características de un neumoencéfalo a tensión provocando una clínica neurológica severa. Presentamos el caso de una paciente de 66 años portadora de una doble válvula de derivación ventrículo-peritoneal que acude a Urgencias con un cuadro de hipertensión intracraneal y que tras la exploración física y la tomografía computarizada se detecta una herida en cuero cabelludo y un neumoencéfalo a tensión. Este defecto en piel se hallaba justo en la zona del borde de una plastia craneal colocada tras la resección de un meningoma parasagital 8 años antes. Se realizó drenaje, reparación de la solución de continuidad y modificación de los factores que agravaban el cuadro del neumoencéfalo (la presencia de unas válvulas de derivación con presiones de salida muy bajas).

La presencia de un neumoencéfalo a tensión debe hacernos pensar en encontrar no sólo el punto de acceso del aire al interior del cráneo sino también las causas que han favorecido que el neumoencéfalo adopte características de alta presión (sistemas de derivación o fístulas de LCR entre otros). De esta forma la orientación terapéutica adecuada tiene que ir destinada a la modificación de estos factores agravantes, además de hacia el drenaje del neumoencéfalo y cierre del defecto craneal.

PALABRAS CLAVE: Neumoencéfalo. Válvula ventrículoperitoneal. Herida en scalp. Hipertensión intracraneal.

Tension pneumocephalus in a patient with a scalp wound and ventriculo-peritoneal shunt: case report and literature review

Recibido: 11-08-08. Aceptado: 15-09-08

\section{Summary}

Skull defects and even meningeal defects are responsible for the majority of pneumocephalus cases. Sometimes, several factors can produce intracranial gas under pressure (tension pneumocephalus) with severe neurological impairment. We present a case of a 66 year old woman with a double ventriculo-peritoneal shunt that was admitted to the emergency service with an intracranial hypertension syndrome. A scalp wound was found on the physical examination and a brain CT showed a tension pneumocephalus. The scalp wound was just located on the border of a cranioplasty made after surgical removal of a parasagital meningioma eight years ago. Evacuation of pneumocephalus, reparation of cranial and meningeal defects and modification of factors contributing to the tension pneumocephalus (ventricular-peritoneal shunts programmed to low pressure) were performed.

When we found a patient with a tension pneumocephalus we must think not only about a skull or meningeal defect but also look for other elements that produce gas inside skull under pressure (shunts, cerebrospinal fluid leak between others). Therefore, therapeutic approach has three parts: pneumocephalus drainage, to find and repair entrance of gas and to modify factors that turn pneumocephalus in a tension pneumocephalus.

KEYWORDS: Pneumocephalus. Ventriculo-peritoneal shunt. Scalp wound. Intracranial hypertension.

Introducción

La entrada de aire en la cavidad craneal recibe el nombre de neumoencéfalo ${ }^{11}$. Esto es relativamente común en diversas circunstancias del mundo de la Neurocirugía y

Abreviaturas. LCR: líquido cefalorraquídeo; NT: neumoencéfalo a tensión; TC: tomografía computarizada; RM: resonancia magnética. 
Tabla 1

Causas de neumoencéfalo

Yatrogénica:

- Postquirúrgica:

- Craniotomía (especialmente con el paciente sentado).

- Agujeros de trepano usados sobre todo en la evacuación de subdurales crónicos.

- Cirugía hipofisaria.

- Inserción de sistemas de derivación del líquido cefalorraquídeo.

- Cirugía en ORL (senos paranasales, oído medio...).

- Tras procedimientos invasivos:

- Punción lumbar.
- Anestesia espinal.
- Ventriculostomía

- Uso de óxido nitroso en anestesia con dura abierta.

Tumoral por invasión ósea (osteomas, epidermoides, tumores hipofisarios...):

- Por invasión craneal

- Por invasión de cavidades neumatizadas (senos, mastoides, oído medio y externo...)

Postraumática:

- Fracturas que afectan a cavidades neumatizadas como los senos.

- Fracturas de la convexidad con aperturas durales.

Otras:

- Microorganismos productores de gas.

- Barotrauma durante submarinismo.

- Espontáneo

de la Otorrinolaringología. Tal es el caso de cirugías de la base de cráneo $^{7}$, hipofisaria ${ }^{23}$ paranasal $^{4}$ y del oído medio ${ }^{6,17}$; evacuación de hematomas subdurales $\operatorname{crónicos}^{2,10}$; tras un trauma craneal ${ }^{18}$; en relación con microorganismos productores de gas $^{19,25}$; asociados a tumores que invaden senos $^{9,16,24}$; en portadores de válvulas de derivación ${ }^{21} \mathrm{e}$ incluso de forma espontánea ${ }^{5,12}$ (tabla 1 ). Dado que no existen series grandes es muy difícil saber cuál es la frecuencia de cada proceso etiológico ${ }^{13}$.

La mayoría de las veces cursa de forma asintomática y se resuelve espontáneamente ${ }^{3,13,14}$. En otras ocasiones progresa y puede manifestarse clínicamente llegando incluso a desarrollar un deterioro neurológico del paciente por hipertensión intracraneal o efecto masa y recibiendo el nombre de neumoencéfalo a tensión (NT).

En este trabajo presentamos un caso complejo de neumoencéfalo a tensión tras una solución de continuidad en cuero cabelludo no traumática, provocado por la presencia de un sistema de derivación ventrículo-peritoneal. Así mismo, revisamos la fisiopatología de este proceso, la discriminación mediante técnicas de imagen entre un neumoencéfalo a tensión o sin tensión y las soluciones terapéuticas posibles.

\section{Caso clínico}

Mujer de 66 años que acude a urgencias acompañada de sus familiares por un cuadro clínico de vómitos, cefalea, bradipsiquia, somnolencia y alteración de la marcha y del lenguaje agudizado en las últimas 24 horas pero de 5 días de evolución.

Ocho años antes fue intervenida de un meningioma parasagital derecho desarrollando un quiste porencefálico a tensión que obligó a la evacuación y retirada del cráneo con posterior colocación de plastia sintética. Dada la ausencia de mejoría se colocaron en otro centro dos válvulas de derivación una cisto-peritoneal y la otra ventrículo-peritoneal a una presión de apertura de $6 \mathrm{~cm}$ de $\mathrm{H} 2 \mathrm{O}$. Tras dicha colocación la paciente mejoró y permaneció con una hemiparesia izquierda residual que había mejorado hasta permitir la deambulación progresiva.

Al ingreso la paciente presentaba un Glasgow de 11 


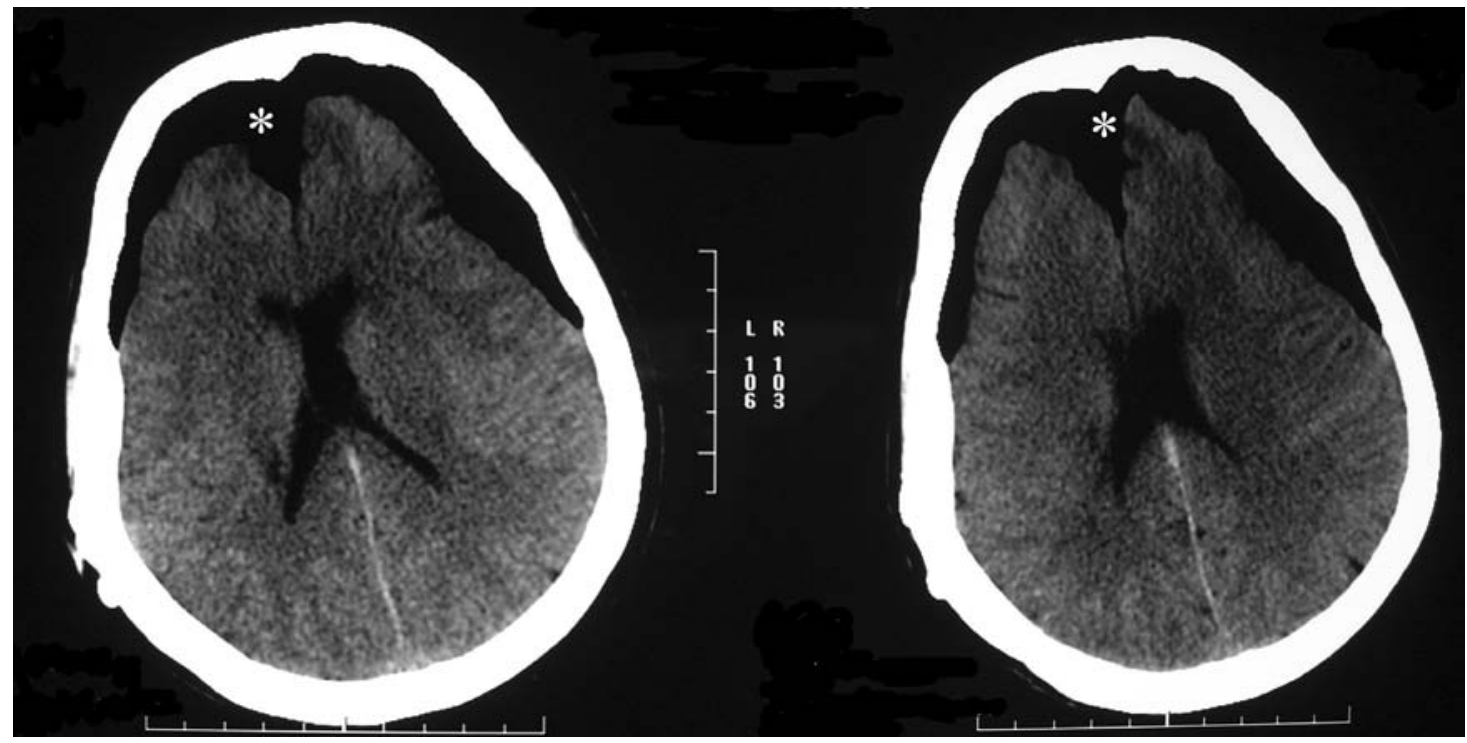

Figura 1: TC craneal el día de ingreso de la paciente que muestra un gran neumoencéfalo subdural bifrontal con efecto masa sobre las astas frontales ventriculares y con el signo del Monte Fuji presente marcado con un asterisco de color blanco.

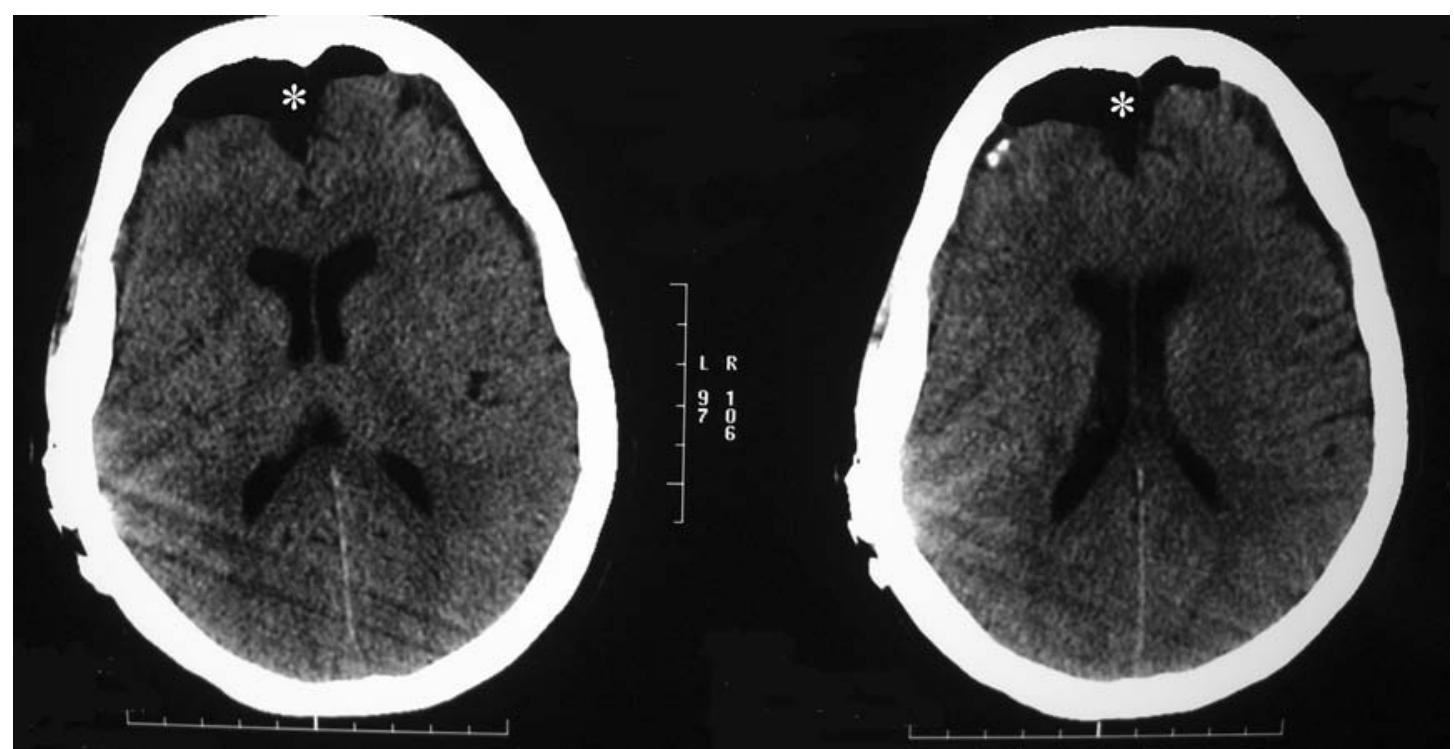

Figura 2. TC de control 72 horas después del drenaje del neumoencéfalo que muestra la disminución de tamaño del neumoencéfalo y la ausencia de efecto masa del mismo.

(O3, V3, M5) con la paresia en el hemicuerpo izquierdo ya conocida. No presentaba alteración en pares craneales. Dados los antecedentes de la paciente y la disminución del nivel de conciencia se hizo una tomografía computarizada (TC) craneal en la que se visualizaba un gran neumoencéfalo a tensión fronto-temporo-parietal bilateral con obliteración de los espacios subaracnoideos y ventriculares. El signo del Monte Fuji, consistente en una separación y compresión de los lóbulos frontales con un ensanchamiento del espacio interhemisférico y subdural dando la punta de ambos lóbulos la apariencia de dicho monte, estaba presente ${ }^{15}$ (Fig 1). Además de dicho hallazgo, la TC informó de otros hallaz- gos conocidos como eran la craneotomía fronto-parietal derecha además de la cavidad porencefálica y la presencia de una doble válvula de derivación. Ante la presencia de aire intracraneal y la ausencia de trauma previo se buscó una solución de continuidad que justificara la entrada de dicho aire. Se observo una herida en cuero cabelludo oculta por el pelo asomando por ella un borde de la plastia que la familia refería no haber visto hasta entonces. Se decidió reprogramar la válvula a una presión de apertura mayor (10 cm de H20), evacuar el neumoencéfalo y reparar la solución de continuidad modelando la plastia craneal. Se realizó un trépano, observándose una salida de aire a pre- 


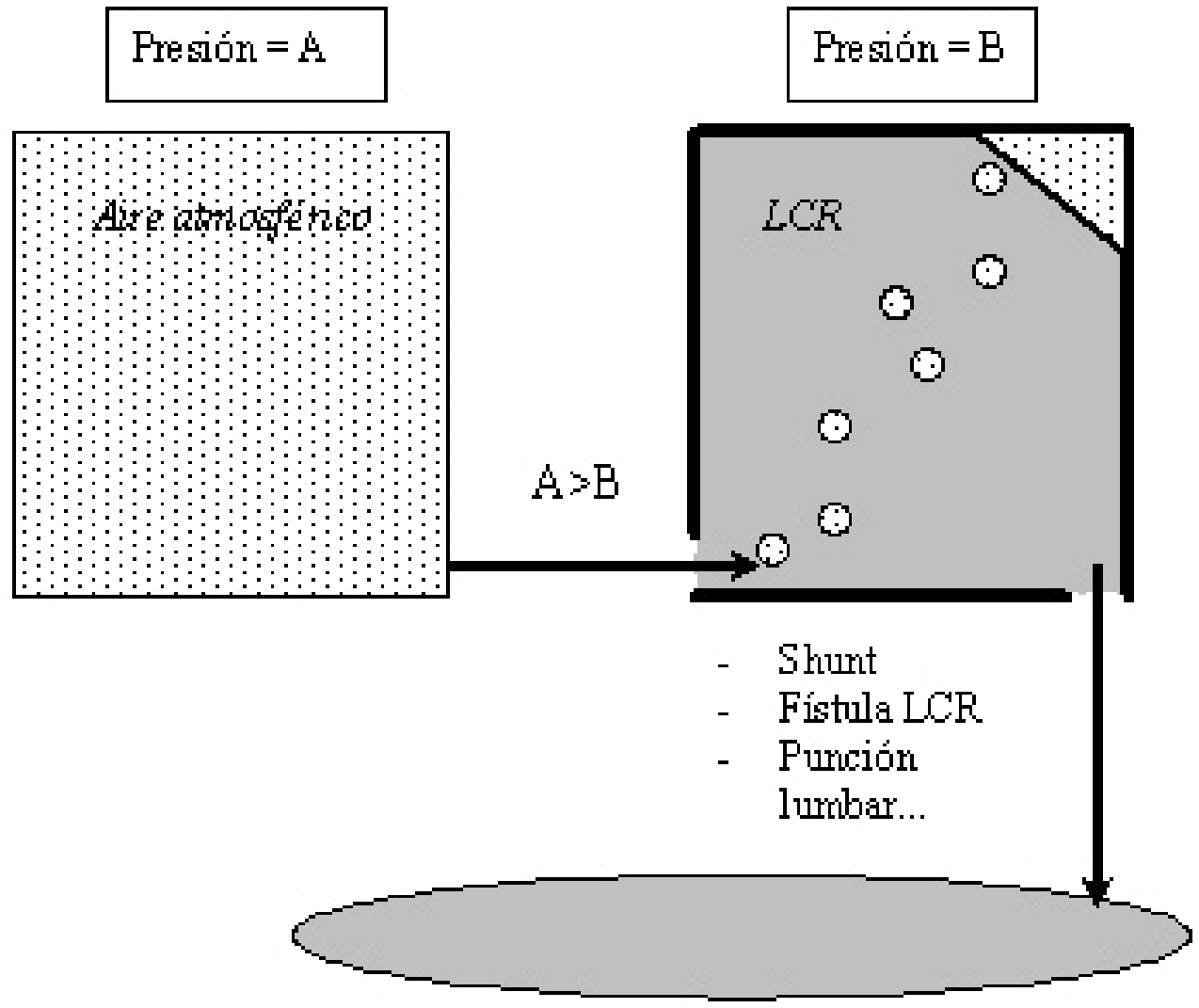

Esquema 1. La presión A representa la presión del aire atmosférico. La presión B representa la presión intracraneal. Cuando existe una solución de continuidad a nivel craneal si la presión de A es mayor que la de B el aire puede penetrar dentro del cráneo. Esta situación se puede ver agravada por situaciones en las que existe una pérdida de LCR por shunts, drenajes lumbares o fístulas ya que a la disminución de la presión que estas situaciones provocan en el compartimento B hay que sumar el espacio libre que ahora se ocupa por aire en lugar de por LCR.

sión, y se dejó un drenaje conectado a un sistema cerrado. Una vez evacuado el neumoencéfalo, pudo repararse el defecto de piel eliminando tejidos no viables tanto óseos como dérmicos y cerrarse en primera intención al no observarse signos de infección local ni sistémicos. La paciente pasó a Reanimación donde mejoró del nivel de conciencia. Se instauró medidas profilácticas antibióticas con una cefalosporina de $3^{\text {a }}$ Generación. Dada la mejora clínica fue dada de alta de Reanimación a las 48 horas de la cirugía con un Glasgow de 15 y sin ningún déficit añadido a su hemiparesia ya conocida. En planta se mantuvo afebril, con cefalea opresiva frontal que fue mejorando con el paso de los días. El drenaje se retiró 72 horas después de la cirugía tras una TC de control en el que se evidenciaba la mejoraba del neumoencéfalo (Fig 2). Tras la retirada del drenaje se mantuvo una semana ingresada a la paciente con tratamiento médico y rehabilitador. Se tuvo especial cuidado de la herida y de cualquier signo de infección (osteomielitis o meningitis). A los 10 días de la intervención se retiraron los puntos de sutura. La paciente mejoró clínicamente hasta poder deambular de nuevo con ayuda, cediendo casi por completo la cefalea. $\mathrm{Al}$ alta hospitalaria la paciente se encontraba en su estado basal.

\section{Discusión}

Para que se produzca un neumoencéfalo debe existir generalmente una solución de continuidad a través del cráneo e incluso de las meninges ya que el aire puede encontrarse no sólo epidural sino también subdural, subaracnoideo, intraparenquimatoso o intraventricular. La entrada de aire puede deberse al simple hecho de la apertura craneal y meníngea durante ciertas intervenciones quirúrgicas con el posterior cerrado hermético y la consiguiente retención de aire intracraneal. En muchas ocasiones, sin embargo, la causa así como la cantidad de aire introducido depende de un gradiente de presiones. De esta forma, en presencia de una solución de continuidad, cuando la presión dentro del compartimento craneal es menor que la atmosférica se produce dicha entrada de aire. Por tanto, existen dos tipos de factores que pueden agravar el cuadro. Por un lado están aquéllos que reducen la presión intracraneal como 
son: la salida de líquido cefalorraquídeo por maniobras como el Valsalva y los cambios posturales, los drenajes lumbares o ventrículo-peritoneales y la deshidratación o la reducción de masa tumoral por radioterapia o radiocirugía. Por otro lado tenemos aquellos factores que aumentan la presión del aire extradural: la apnea obstructiva del sueño y la ventilación a presión positiva, un barotrauma ocasionado durante submarinismo, etc.

Además de este gradiente de presiones, se han descrito otros procesos que pueden justificar la entrada de aire intracraneal. De esta forma en el caso de las fístulas de líquido cefalorraquídeo se han sugerido dos modelos: el de la botella de gaseosa invertida donde la salida de LCR favorece la entrada de aire en forma de burbujas reemplazando el espacio de este líquido; o el modelo de válvula unidireccional donde es la forma de la fístula en contacto con las estructuras adyacentes la que permite la entrada de aire pero impide su salida ${ }^{26}$.

Otros mecanismos que pueden causar la entrada de aire intracraneal sin relación con los anteriores son la presencia de microorganismos productores de gas ${ }^{19,25}$, el uso del gas nitroso como anestésico con aperturas durale ${ }^{20} \mathrm{o}$ incluso la ley de Charles-Gay Lussac ${ }^{1}$ que habla de la expansión de gases al aumentar al temperatura.

La perpetuación de estos mecanismos así como su combinación puede dar lugar a que el neumoencéfalo se haga a tensión provocando clínica de hipertensión intracraneal y poniendo en riesgo la vida del paciente.

De todos los mecanismos anteriores descritos, en nuestro caso, el neumoencéfalo a tensión fue debido a un gradiente de presiones a través de una solución de continuidad no traumática en la piel. Esto permitía al aire atmosférico entrar y provocar el neumoencéfalo. Si la paciente no hubiera tenido la doble válvula de derivación probablemente este neumoencéfalo nunca hubiera tenido las características de tensión y tampoco hubiera dado la clínica que desarrollaba (esquema 1). Este neumoencéfalo todavía se autoperpetuaba más por el hecho de tener las válvulas a presiones de apertura muy bajas.

El neumoencéfalo puede cursar de forma asintomática o con una clínica vaga e inespecífica ${ }^{13}$. Algunos síntomas pueden ser cefalea, náuseas y vómitos, letargia, alteraciones del nivel de conciencia y, en ocasiones, meningismo. Pueden presentar de forma rara crisis convulsivas ${ }^{14}$. Cuando se desarrolla un neumoencéfalo a tensión por alguno de los mecanismos mencionados anteriormente la clínica puede ser de focalidad neurológica o de aumento de la presión intracraneal

El tratamiento para el neumoencéfalo la mayoría de las veces es la observación clínica y radiológica. La opción quirúrgica se suele reservar para aquellas ocasiones en las que el neumoencéfalo se convierte en a tensión, cuando éste presenta como causa desencadenante una fístula de líquido o en aquellos neumoencéfalos muy sintomáticos. El tratamiento entonces pasa por tres actuaciones y establecer el orden correcto de esas tres actuaciones. La primera de ellas es encontrar el punto o solución de continuidad por donde entra el aire y en caso de haber fístula de líquido repararla. La segunda es evacuar el neumoencéfalo que empieza a hacer efecto masa. La tercera es averiguar si existe un factor agravante que actúe sobre el mismo y procurar, en la medida de lo posible, ponerle solución (ventilación a presión positiva o ajustar presión de apertura en válvulas programables, etc.). Se discute si es conveniente el drenaje del neumoencéfalo previo a la reparación de la solución de continuidad ya que algunos autores ${ }^{8}$ piensan que en el caso de hacerse, la disminución de la presión intracraneal podría hacer que se produjera una entrada aún mayor de aire. Sin embargo, la clínica del paciente hace que en muchos casos sea necesario el drenaje del neumoencéfalo a tensión previo a la búsqueda o reparación de la solución de continuidad ${ }^{22}$.

En nuestro caso, la paciente presentaba una solución de continuidad visible en cuero cabelludo provocado por la acción de uno de los bordes de la plastia craneal sobre una piel debilitada por cirugías previas. La plastia se hallaba parcialmente levantada por la presión que ejercía el neumoencéfalo con lo que aun más se perpetuaba el efecto del borde de la plastia sobre la herida. La técnica usada para solucionar el problema pasaba por los tres puntos antes mencionados: reparación del defecto, drenaje del neumoencéfalo y evitar los factores agravantes, en este caso la presencia de dos válvulas de derivación con presiones de apertura muy baja (6 $\mathrm{cm}$ de H20). Lo primero que se hizo fue el drenaje del neumoencéfalo ya que era imposible la recolocación y reparación de la plastia craneal debido a la elevación que presentaba uno de sus bordes por el aumento de la presión intracraneal. Una vez drenado el neumoencéfalo por medio de un agujero de trépano y con la salida de aire a presión, se colocó un drenaje, se conectó a un sistema cerrado y se cerró de forma hermética la apertura de piel necesaria para el trépano de drenaje. A continuación se pudo reparar el defecto craneal, así como la piel, eliminando las estructuras más necróticas o menos viables, tanto de hueso como de cuero cabelludo. Aunque en este caso se hizo de forma previa a la cirugía que acabamos de comentar, se elevó la presión de apertura de las válvulas para evitar el hiperdrenaje que favorece la pérdida de líquido y la entrada, por tanto, del aire a través de la herida.

\section{Conclusión}

La presencia de aire intracraneal nos tiene que llevar a pensar la mayoría de las veces en la presencia de una solución de continuidad, muchas veces conocida pero otras no tanto. Una vez encontrada dicha solución de continuidad 
es necesario la reparación de la misma y la valoración del drenaje del neumoencéfalo en función de las características de éste y de la clínica que esté provocando al paciente. Conviene destacar que en ocasiones, como ha sido nuestro caso, es necesario descartar la presencia de ciertos factores agravantes como son la presencia de sistemas de derivación del LCR, y, en el caso de existir, valorar cómo repercuten en la fisiopatología del neumoencéfalo de cada paciente.

\section{Bibliografía}

1. Black, P.M., Davis, J.M., Kjellberg, R.N., Davis K.R.: Tension pneumocephalus of the cranial subdural space: a case report. Neurosurgery $1979 ; 5: 368-370$.

2. Bremer, A.M., Nguyen, T.Q.: Tension pneumocephalus after surgical treatment of chronic subdural hematoma: report of three cases. Neurosurgery 1982; 11: 284-287.

3. Dandy, W.E.: Pneumocephalus (intracranial pneumatocele or aeorcele). Arch Surg 1926; 12: 949-982.

4. Davis, D.H., Laws, E.W.Jr, McDonald, T.J., Salassa, J.R., Phillips, L.H $2^{\text {nd }}$.: Intraventricular tension pneumocephalus as a complication of paranasal sinus surgery: case report. Neurosurgery 1982; 8: 574-576.

5. Dowd, G.C., Molony, T.B., Voorhies, R.M.: Spontaneous otogenic pneumocephalus. Case report and review of the literature. J Neurosurg 1998; 89: 1036-1039.

6. Finsnes, K.A.: Lethal intracranial complication following air insufflation with a pneumatic otoscope. Acta Otolaryngol 1973; 75: 436-438.

7. Fliss, D.M., Gil, Z., Spektor, S et al.: Skull base reconstruction after anterior subcranial tumor resection. Neurosurg Focus. 2002; 12: e10.

8. Haran, R.P., Chandy, M.S.: Symtomatic pneumocephalus after transsphenoidal surgery. Surg neurol 1997; 48: 575-578.

9. Hirst, L.W., Miller, N.R., Hodges, F.J $3^{\text {rd }}$., Corbett, J.J., Thomspson, S.: Sphenoid pneumosinus dilatans. A sign of meningioma originating in the optic canal. Neuroradiology 1982; 22: 207-210.

10. Ishiwata, Y., Fujitsu, K., Sekino, T., et al.: Subdural tension pneumocephalus following surgery for chronic subdural hematoma. J Neurosurg 1988; 68: 58-61.

11. Jelsma, F., Moore, D.F. Craneal aerocele. Am J Surg 1954; 87: 437-451.

12. Krayenbühl, N., Alkadhi, H., Jung, H.H., Yonekawa, Y.: Spontaneous otogenic intracerebral pneumocephalus: case report and review of the literature. Eur Arch Otorhinolaryngol. 2005; 262: 135-138

13. Markham, J.W.: The clinical features of pneumocephalus based upon a survey of 284 cases with report of 11 additional cases. Acta Neurochir (Wien). 1967; 16: 1-78.

14. Mauri-Llerda, J.A., Tejero-Juste, C., Dolz-Aspas, R.: Neumoencefalo y crisis epilépticas agudas sintomáticas. Rev
Neurol 2007; 44: 126-127.

15. Michel, S.J.: The Mount Fuji sign. Radiology 2004; 232: 449-450.

16. Miller, N.R., Golnik, K.C., Zeidman, S.M., North, R.B.: Pneumosinus dilatans: a sign of intracranial meningioma. Surg Neurol 1996; 46: 471-474.

17. Nagai, H., Moritake, K.: Otogenic tension pneumocephalus complicated by eustachian tube insufflation in a patient with a ventriculoperitoneal shunt. Case report. J Neurosurg. 2007; 106: 1098-1101.

18. Oge, K., Akpinar, G., Bertan, V.: Traumatic subdural pneumocephalus causing rise in intracranial pressure in the early phase of head trauma: report of two cases. Acta Neurochir (Wien) 1998; 140: 655-658.

19. Penrose-Stevens, A., Ibrahim, A., Redfern, R.M.: Localized pneumocephalus caused by Clostridium perfringens meningitis. Br J Neurosurg 1999; 13: 85-86.

20. Raggio, J.F., Fleischer, A.S., Sung, Y.F., Hoffman, J.C..: Expanding pneumocephalus due to nitrous oxide anesthesia: case report. Neurosurgery 1979; 4: 261-263.

21. Ruge, J.R., Cerullo, L.J., McLone, D.G.: Pneumocephalus in patients with CSF shunts. J Neurosurg. 1985; 63: 532-536.

22. Ruiz-Juretschke, F., Mateo-Sierra, O., Iza Vallejo, B., Carrillo-Yagüe, R.: Neumoencéfalo intraventricular a tensión secundario a cirugía transesfenoidal: presentación de un caso y revisión de la literatura. Neurocirugía 2007; 18 : 134-137.

23. Sawka, A.M., Aniszewsski, J.P., Young, W.F., Nippoldt, T.B., Yanez, P., Ebersold, M.J.: Tension pneumocranium, a rare complication of transphenoidal surgery: Mayo Clinic experience 1976-1998. J Clin Endocrin Met 1999; 84: 4731- 4734.

24. Scholsem, M., Collignon, F., Deprez, M., Martin, D.: Spontaneous pneumocephalus caused by the association of pneumosinus dilatans and meningioma. Case illustration. J Neurosurg 2006; 105: 934.

25. Tanaka, T., Takagi, D., Takeyama, N., Kitazawa, Y.: "Spontaneous" pneumocephalus associated with aerobic bacteremia. Clin Imaging 1989; 13: 134-139.

26. Walker, F.O., Vern, B.A.: The mechanism of pneumocephalus formation in patients with CSF fistulas. J Neurol Neurosurg Psychiatry. 1986 Feb; 49: 203-205

González-Bonet, L.G.; Goig-Revert, F.A.; RodríguezMena, R.; Barcia-Mariño, C.: Neumoencefalo a tensión tras herida en cuero cabelludo en portadora de válvula de derivación ventrículo-peritoneal: caso clínico y revisión de la literatura. Neurocirugía 2009; 20: 152-158.

Correspondencia postal: Servicio de Neurocirugía. Hospital General Universitario de Valencia. Avda. Tres Cruces, 2. Valencia, 46014, Spain. 
Comentario al trabajo Neumoencéfalo a tensión tras herida en cuero cabelludo en portadora de válvula de derivación ventrículo-peritoneal: caso clínico y revisión de la literatura de González-Bonet y cols.

Los autores presentan un caso de neumoencéfalo a tensión en una paciente portadora de una craneoplastia acrílica que se fue desplazando desde su posición original hasta crear una dehiscencia cutánea a través de la cual penetró el aire al interior del cráneo, hecho favorecido por la situación de hipopresión relativa generada por la coexistencia de una derivación ventricular y otra cistoperitoneal de LCR.

El desarrollo de neumoencéfalo tras la implantación de una derivación es un fenómeno conocido hace tiempo ${ }^{5}$, aunque se trata de un fenómeno relativamente infrecuente: Villarejo y cols..$^{10}$ revisaron 12 casos en 1998 mientras que Ugarriza y cols. ${ }^{9}$ publicaron en 2001 tres casos más y una revisión de la literatura donde sólo encontraron 37 casos descritos. La entrada del aire al espacio intracraneal suele producirse a través de un defecto en la base del cráneo ${ }^{4,5,9,10}$ aunque otras vías de acceso han sido descritas: desde el extremo abdominal de la derivación ${ }^{3,7}$, tras la realización de una craneotomía -particularmente en la fosa posterior y en posición sentada- ${ }^{1,6}$, o debido a la presencia patológica de aire en relación a la derivación como la documentada en casos de enfisema subcutáneo o fístula auriculobronquial ${ }^{2,8}$.

El presente caso describe una nueva vía de acceso, proporcionada por la dehiscencia cutánea producida por una craneoplastia. Los autores decidieron mantener este cuerpo extraño tras realizar únicamente una limpieza local del material expuesto, si bien estrecharon el seguimiento clínico y radiológico para detectar una posible infección de la plastia. La evolución a largo plazo permitirá conocer si este planteamiento evita el desarrollo de infección y resuelve el problema mecánico originado por la plastia.

El incremento en el número de derivaciones ventriculares de LCR implantadas puede favorecer que la incidencia de esta complicación relativamente infrecuente aumente en los próximos años. Las derivaciones programables pueden modular la hipopresión inducida por la derivación pero no resuelven el problema del neumoencéfalo si no se localiza y soluciona el punto de entrada del aire. No obstante, pueden ser útiles en la prevención del neumoencéfalo en situaciones controladas: en nuestra experiencia, la regulación al alza de la presión de apertura de las derivaciones programables durante la cirugía sobre la fosa posterior ha disminuido de forma notable la cantidad y la duración del neumoencéfalo posquirúrgico que característicamente acompaña a este abordaje y que a menudo complica el postoperatorio con somnolencia, cefalea, vómitos, focalidad neurológica transitoria e incluso disfunción valvular. Obviamente, las válvulas on-off también son útiles para este cometido aunque la posibilidad de "apagado" accidental de la derivación probablemente han limitado la generalización en el uso de estos sistemas.

\section{Bibliografía}

1. Hernández-Palazón, J., Sánchez-Bautista, S., Tortosa Serrano, J.A., Mulero Cervantes, J.F., Martínez-Lage, J.F.: Pressure pneumocephalus after posterior fossa surgery in the sitting position . Rev Esp Anestesiol Reanim. 1998 Feb; 45: 68-71.

2. Horton, D.D., Pollay, M., Reynolds, A.F. Jr.: Intraventricular pneumocephalus secondary to subcutaneous emphysema: a case report. Neurosurgery. 1984 Oct; 15: 557558.

3. Jea, A., Baskaya, M.K., Farhat, H., Benglis, D., Zauner, A.: Pneumocephalus in a patient with a ventriculoperitoneal shunt after percutaneous gastrojejunostomy catheter placement: case report. Surg Neurol. 2006 Jan; 65: 87-89.

4. Nagai, H., Moritake, K.: Otogenic tension pneumocephalus complicated by eustachian tube insufflation in a patient with a ventriculoperitoneal shunt. Case report. J Neurosurg. 2007 Jun; 106: 1098-10101.

5. Pitts, L.H., Wilson, C.B., Dedo, H.H., Weyand, R.: Pneumocephalus following ventriculoperitoneal shunt. Case report. J Neurosurg. 1975 Nov; 43: 631-633.

6. Por, Y.C., Barcelo, C.R., Salyer, K.E.: Extradural tension pneumocephalus after posterior cranial vault remodeling for ventriculoperitoneal shunt-induced craniosynostosis. J Craniofac Surg. 2005 May; 16: 504-508.

7. Shetty, P.G., Fatterpekar, G.M., Sahani, D.V., Shroff, M.M.: Pneumocephalus secondary to colonic perforation by ventriculoperitoneal shunt catheter. Br J Radiol. 1999 Jul; 72: 704-705.

8. Stuntz, J.T., Shuman, R.M.: Pneumocephalus secondary to cerebrobronchial fistula formed by a ventriculoatrial shunt. Case report. J Neurosurg. 1977 Aug; 47: 286-289.

9. Ugarriza, L.F., Cabezudo, J.M., Lorenzana, L.M., Porras, L.F., García-Yagüe, L.M.: Delayed pneumocephalus in shunted patients. Report of three cases and review of the literature. Br J Neurosurg. 2001 Apr; 15: 161-167.

10. Villarejo, F., Carceller, F., Alvarez, C., Bencosme, J., Pérez Díaz, C., Goldman, L., Pascual, A.: Pneumocephalus after shunting for hydrocephalus. Childs Nerv Syst. 1998 Jul; 14: 333-337.

P. Miranda. Valencia 